\title{
GENETIC ALGORITHMS FOR DESIGN OF PIPE NETWORK SYSTEMS
}

\author{
Hong-Min Shau \\ Doctor Candidate, Department of Harbor and River Engineering, National Taiwan Ocean University. Keelung, Taiwan \\ 202, R.O.C \\ Bi-Liang Lin \\ Assistant Professor, Department of Civil Engineering, Ming Hsin University of Science and Technology, Hsinchu, \\ Taiwan 304, R.O.C \\ Wen-Chih Huang \\ Professor, Department of Harbor and River Engineering, National Taiwan Ocean University, Keelung, Taiwan 202, \\ R.O.C., huangwc@mail.ntou.edu.tw
}

Follow this and additional works at: https://jmstt.ntou.edu.tw/journal

Part of the Civil and Environmental Engineering Commons

\section{Recommended Citation}

Shau, Hong-Min; Lin, Bi-Liang; and Huang, Wen-Chih (2005) "GENETIC ALGORITHMS FOR DESIGN OF PIPE NETWORK SYSTEMS," Journal of Marine Science and Technology. Vol. 13: Iss. 2, Article 7.

DOI: $10.51400 / 2709-6998.2112$

Available at: https://jmstt.ntou.edu.tw/journal/vol13/iss2/7

This Research Article is brought to you for free and open access by Journal of Marine Science and Technology. It has been accepted for inclusion in Journal of Marine Science and Technology by an authorized editor of Journal of Marine Science and Technology. 


\title{
GENETIC ALGORITHMS FOR DESIGN OF PIPE NETWORK SYSTEMS
}

\author{
Hong-Min Shau*, Bi-Liang Lin**, and Wen-Chih Huang***
}

Key words: genetic algorithm, pipe network systems, optimal design, practical requirement.

\begin{abstract}
In the last three decades, a significant number of methods for optimal design of pipe network systems have been developed using linear programming, non-linear programming, dynamic programming, enumeration techniques, and genetic algorithm (GA). This paper presents a genetic algorithm (GA) approach to the design of a pipe network systems. The objectives considered are minimization of the network cost in the practical requirement. Of all the preceding methods, GA based methods appear to be robust, as they can handle discrete pipes sizes with ease and produce a set of alternatives. By judgmentally selecting the Ruey-Fang district water supply system for the case study and by comparing the data gathered from the case study, this study also aims to verify the efficacy of the proposed method. The finding indicates that the proposed method is superior to enumeration techniques with respect to solution speed and cost.
\end{abstract}

\section{INTRODUCTION}

Nowadays, designers often make use of commercial simulation packages such as KYPIPE, WATER, CYBERNET, EPANET2.0, WATERCAD and MIKENET [8]. The trial and error process is repeated until satisfactory alternatives are found. Users obtain at best near-optimal solutions. A number of researchers have used linear programming (Morgan and Goulter [10]; Fujiwara [3]; Kessler and Shamir [6]; Wu and Lin [14]) or nonlinear programming (Liebman et al. [8]; Brooke et al. [1]; Lansey and Mays [7]) to obtain an optimal design of a pipe network. In general, nonlinear programming packages use a constrained generalized

Paper Submitted 06/12/04, Accepted 07/21/04. Author for Correspondence: Wen-Chih Huang. E-mail: huangwc@mail.ntou.edu.tw.

*Doctor Candidate, Department of Harbor and River Engineering, National Taiwan Ocean University. Keelung, Taiwan 202, R.O.C.

**Assistant Professor, Department of Civil Engineering, Ming Hsin University of Science and Technology, Hsinchu, Taiwan 304, R.O.C.

***Professor, Department of Harbor and River Engineering, National Taiwan Ocean University, Keelung, Taiwan 202, R.O.C. reduced gradient (GRG) technique to identify a local optimal solution. The limitations of using linear and nonlinear programming packages to solve a real life complex pipe system include:

(1) The solution may specify sizes that are not commercial diameters, and therefore necessitates rounding of final solution.

(2) The solution found by these optimal techniques is at most an optimal solution, not the global optimal solution.

(3) The size of network design depends on the number of constraints. A specific software may be used for the decision of the size.

Numerous researchers have proposed different optimal designs of pipe network systems as a combinatorial optimization problem and solved with enumeration and genetic algorithm (Gessler [4]; Lin et al. [9]; Simpson et al. [13]; Dandy et al. [2]; Savic and Walters [12]).

With respect to preliminary solutions to optimization problems of the network system of water supply, Goldberg [5], proposed well-founded frameworks. In principle, the GA is regarded a search procedure for the minimum or maximum of an unconstrained function by using random selection processes, which simulate reproduction, selection, crossover, and mutation of living creatures.

Pilar et al. [11] proposed a convergence optimization to modify the simple GA. They tried to explain how species are selected to change and how they are transformed into different species. The GA is regarded a search procedure applicable to different problems for optimal solutions. To meet the needs of nodes and the layout of hydraulic elements, the optimal design of a looped network for gravity systems is decided by the set of pipe size, which results in the minimum investment cost.

Simpson et al. [13] applied the GA to solve the pipe network systems, and compared it with the complete enumeration method and the nonlinear programming. Dandy et al. [2] improved Simpson's efficiency by applying the simple GA directly. They also 
compared the problems of New York tunnels with those of previous research documents. The result showed that the GA could generate a lower-cost design program than the methods previously applied.

Based on the document analysis, this research applied the GA to the optimal design of the pipe network systems. This study aims at exploring how to obtain optimal design of the pipe network systems with limited resources and insufficient time. The results of this study are also compared with those of previous studies.

\section{METHODOLOGY}

Advanced by John Holland in the 1970s, the term "Genetic Algorithm" (GA) originated in the Darwinian notion of "Survival of the fittest in natural selection." Ever since its introduction, the GA has successfully solved the discontinuous, the non-differentiable, the non-convex, and the multiple peaks function optimization problems which traditional analytical and numerical optimization method failed to solve [5]. Although the pipe network systems is very complicated, its diameter variables can be easily coded. Over the past years scholars have proposed utilizing the GA to solve pipe network systems optimization.

In a fixed pipe network systems, each pipe and its auxiliary facilities may be regarded as a stage. In the process of design, there are various commercial pipe sizes available for selection. Therefore, the design of the water distribution system is used to precede network simulation under different loading demands as well as to select varied combinations of substitute elements in order to obtain the pressure distribution of the system and gain the water head of each node. To comply with both theoretical and practical needs, the researchers established the optimal mode of the water distribution system planning and design, coupled with each water distribution element. The optimal model is shown below:

$$
\begin{aligned}
& \text { Maximized Fitness }=\frac{1}{\text { Cost }} \\
& \text { Cost }=\sum_{i=1}^{n} \sum_{j=1}^{m_{i}} F_{i}\left(D_{i j}\right) L_{i} X_{i j}+\text { penalty }_{i}
\end{aligned}
$$

Subject to $\sum_{k} Q_{\text {in }}-\sum_{k} Q_{\text {out }}=Q_{k}, k \in$ node

$\sum_{l} h_{l}-D H_{l}=0, l \in$ loop

$$
\begin{aligned}
& \operatorname{HMIN}_{k} \leq H_{k} \leq \operatorname{HMAX}_{k}, k \in \text { node } \\
& \operatorname{VMIN}_{i} \leq V_{i} \leq \operatorname{VMAX} X_{i}, i=1,2, \ldots ., n \\
& X_{i j}=0,1, i=1,2, . ., n \text { and } j=1,2, \ldots \ldots, m_{i}
\end{aligned}
$$

$$
\begin{aligned}
& \sum_{j=1}^{m_{i}} X_{i j}=1, \quad i=1,2, . ., n \text { and } j=1,2, \ldots, m_{i} \\
& \text { and } L_{i}, D_{i} \geq 0
\end{aligned}
$$

Where $D_{i}=$ the diameter in stage $i ; L_{i}=$ the pipe length in stage $i ; n=$ the number of potential pipes in the network; $m_{i}=$ the number of options in the ith stage; $F_{i}\left(D_{i j}\right)=$ the pipes (link) cost function as a function of the diameter, $D_{i j}$; $D_{i j}=$ the diameter of option $j$ at stage $i$; penalty $_{i}=$ the penalty in stage $i ; Q_{i n}=$ the inflow rate at node $k ; Q_{\text {out }}=$ the outflow rate at node $k ; Q_{k}=$ the demand at node $k ; h_{l}=$ the head loss at loop $l ; D H_{l}=$ the difference in water level from the initial node to the last node at loop $l ; H M I N_{k}=$ the minimum head level at node $k ; H M A X_{k}=$ the maximum head level at node $k ; V M I N_{i}=$ the minimum velocity limitation at node $i ; V M A X_{i}=$ the maximum velocity limitation at node $i$; and $X_{i j}=$ the $0-1$ variables for option $j$ in stage $i$.

\section{APPLING THE GA TO THE PIPE NETWORK SYSTEMS DESIGN}

The Improved GA adopted in this study combines the gray coding with the elitist strategy. Compared with the basic GA, the improved GA can increase the probability of finding the global maximum. Figure 1 shows the application of the GA on the flow path of the design of the pipe network systems. The relative parameter used in GA is tested and evaluated for the case the RueyFang water supply system in Taipei County.

\section{The coding of the diameter variables}

If a chromosome is to store correct messages, the

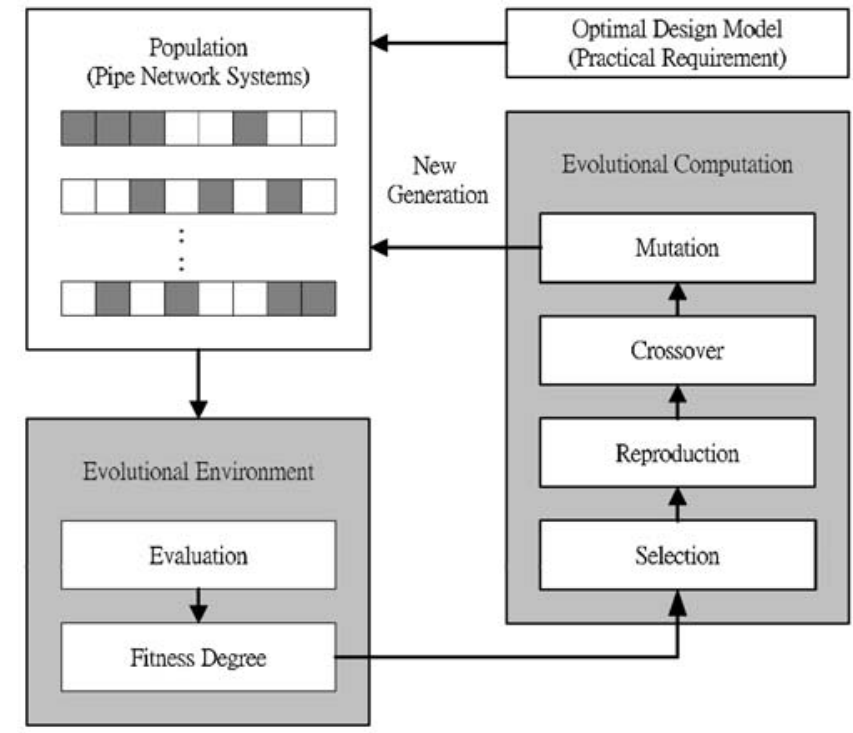

Fig. 1. The flow chart of GA on the design of the pipe network systems. 
gene inside it must have a fixed coding method. In principle, either binary basic coding pattern or gray coding can be applied to diameter coding. By gray code is meant that the set (of numbers) differs from the previous set (of numbers) only in one number. For example, the 011 is differs from the previous 001 only in one number. The corresponding relationship between the diameter variable and the code is shown in Table 1.

\section{Producing the initial population}

Using random sampling produces the initial population. The size of the population depends on the dimension of the pipe network systems. Choosing a suitable population size may enable the researchers to use the GA efficiently and effectively. To comply with the needs of the selected case, the researchers test the numbers from 10 through 1000.

\section{The objective function value evaluation}

Once the initial population is decoded, researchers need to go on with counting the cost of the responding diameter one by one. The sum is the value of objective function as shown in Eq. (1).

\section{Counting the fitness}

The higher the fitness degree is, the better qualities the chromosome possesses, and the more likely they are to be reproduced in the future. With respect to the problem of searching for the maximized objective value, the fitness degree can be directly represented by the objective function. As to the design of the pipe network systems, seeking for the lowest cost is regarded as a problem of the minimized objective function. The function of fitness degree needs to be further treated by using the objective function. This research adopted the reciprocal of the objective function as the fittest degree. The smaller the value of the objective function is, the greater the fittest degree is. If the hydraulic analysis of the system design program does not conform to the design criteria, a penalty factor will be given. An amount of 1,000,000 NT\$ was proposed by this study as the penalty. Eq. (1) is the fitness used in this research.

\section{Reproduction and selection}

By using a predetermined method researchers may select from the present a parent population for matching and reproduction of their offspring. Generally, the roulette wheel method is implemented for the selection of the parent population. This research, however, utilized the tournament selection method. After evenly mixing the parent population, the researchers selected two from it to compare their fitness degree and pick up one that is more likely to evolve. Again, the researchers selected two more from it separately to compare their fitness degree and pick up one that is more likely to evolve. This is to prove that the parent population with better adaptability will further evolve. It is assumed

Table 1. The corresponding relationship chart between the diameter variable and the code

\begin{tabular}{rccccc}
\hline & Diameter & \multicolumn{2}{c}{ Eight pipes } & \multicolumn{2}{c}{ Sixteen pipes } \\
\cline { 3 - 5 } & & Binary coding & Gray coding & Binary coding & Gray coding \\
\hline 1 & 0.10 & 000 & 000 & 0000 & 0000 \\
2 & 0.15 & 001 & 001 & 0001 & 0001 \\
3 & 0.20 & 010 & 011 & 0010 & 0011 \\
4 & 0.25 & 011 & 010 & 0011 & 0010 \\
5 & 0.30 & 100 & 110 & 0100 & 0110 \\
6 & 0.35 & 101 & 111 & 0101 & 0111 \\
7 & 0.40 & 110 & 101 & 0110 & 0101 \\
8 & 0.45 & 111 & 100 & 1000 & 0100 \\
9 & 0.50 & & & 1001 & 1100 \\
10 & 0.60 & & & 1010 & 1101 \\
11 & 0.70 & & & 1011 & 1111 \\
12 & 0.80 & & & 1100 & 1110 \\
13 & 0.90 & & & 1101 & 1010 \\
14 & 1.00 & & & 1110 & 1011 \\
15 & 1.10 & & & 1111 & 1001 \\
16 & 1.20 & & & & 1000 \\
\hline
\end{tabular}


that better genes can therefore be passed on to and inherited by the offspring.

\section{The elitist strategy}

The GA determines the reproduction probability of the offspring's word strings on the basis of fitness degree. However, this does not mean that the word strings of the offspring, which have the optimal fitness, are sure to guarantee reproduction. Therefore, the best individuals may be preset for up-to-now reservation. However, the number of individuals to be reserved depends on practical problems. The researchers use the numbers 100 .

\section{The crossover and the crossover rate}

The selected paired chromosomes produce the offspring by exchanging genes with each other. By means of this, the offspring can possess some of the excellent genes from their parents, and chromosomes with better adaptability are recombined accordingly. Three methods are generally adopted for gene crossover, including single point crossover, dual point crossover, and uniform crossover. This research adopted single point crossover and uniform crossover. The purpose of crossover is to let chromosomes exchange useful information with each other, and get higher fitness in order to have better chromosomes in the next generation and have preferable performance. In case we would like to keep certain specific chromosomes in the next generation, we may adopt crossover rate. The rates of crossover depend on individual problems. Appropriate crossover rate is of significance to quality training. This research set the crossover rate at $0.5,0.6$, or 0.7 .

\section{The mutation}

Mutation aims to obtain more information. However, mutation rates tend to be very small. This is because that if mutation rate is too high, chromosomes might lose some information in the process of mutation. Mutation rate, however, is flexible. If chromosomes are found to cease to progress after a specific period of time, we may increase the mutation rate to enable more changes to take place. In general, two handling methods are adopted:

\section{(1) The gene mutation}

When the occurring random numbers are smaller than the preset mutation rate, the system will randomly choose genes, and change the gene values.

(2) The transformation mutation:

This is used to change the gene's location in a certain section of the chromosome without changing its value. The setting of the mutation rate directly influences the result of the search for excellent individuals. If the value is too small, mutation will become dysfunctional, thus leading to premature convergence. Large mutation rate, on the contrary, may keep the offspring from inheriting good genes from their parent population. The rates of mutation, therefore, depend on the nature of individual problem. Its suitable value is usually obtained by the experiment. In this study, the researchers set the mutation rate at 0.02 . By now, the parent population has evolved into the second generation. By repeating the above-mentioned steps 3 through 8 , we may hope to obtain better variable solutions through generations of mathematical calculations. And if possible, we may even get a solution that is converged on and approximates the optimal solution of the whole.

\section{CASE STUDY AND DISCUSSION}

\section{Practical design cases and computer program}

The Ruey-Fang water supply system in Taipei County used in other studies or reports are selected to illustrate the practical application of the GA. There are 26 pipelines, 20 nodes, and 2 water intake points. One of intake is water treatment plant; the other is the KungLiao system support. Table 2 is the cost per unit length of DIP used in the optimal design of Ruey-Fang system. The Pipe and Node Characteristics for Ruey-Fang system is in Table 3. From operation data, the maximum day of water-consuming amount of the entire district is $15,480 \mathrm{CMD}$, and the minimum day is 5,710 CMD. The Ruey-Fang system hydraulic analysis figure shows in Figure 3. The results and the discussions are provided below. A computer program was developed based on the GA, and written in FORTRAN. The modified Hardy-Cross method is adopted in this study for hydraulic analysis simulation. 0.0001 CMS is used for the water convergence precision at node. The remaining adopted data related are the same as those in the report.

\section{The finding and results}

\section{(1) The population size:}

The larger the system is, the larger the population size should be accordingly. As a result, there will be sufficient points scattered in the solution area. For this reason, 100 populations will suffice for this case study. (2) The result of the solution:

When the selected parameter is suitable, the GA can identify an economical program, which costs much, lower than the case using the enumeration method previously used. This means that the range of the practicable diameter, which is formerly fixed, can be subject 
Table 2. The cost per unit length of DIP used in the optimal design of Ruey-Fang system

\begin{tabular}{rcccc}
\hline No. & $\begin{array}{c}\text { Diameter } \\
(\mathrm{M})\end{array}$ & $\begin{array}{c}\text { Material cost } \\
(\mathrm{NT} / \mathrm{M})\end{array}$ & $\begin{array}{c}\text { Construction cost } \\
(\mathrm{NT} \$ \mathbf{M})\end{array}$ & $\begin{array}{c}\text { Total cost } \\
(\mathrm{NT} / \mathrm{M})\end{array}$ \\
\hline 1 & 0.10 & 516 & 348 & 864 \\
2 & 0.15 & 732 & 427 & 1159 \\
3 & 0.20 & 958 & 514 & 1472 \\
4 & 0.25 & 1088 & 612 & 1700 \\
5 & 0.30 & 1367 & 707 & 2074 \\
6 & 0.35 & 1619 & 1019 & 2638 \\
7 & 0.40 & 2061 & 1051 & 3112 \\
8 & 0.45 & 2468 & 1338 & 3806 \\
9 & 0.50 & 2908 & 1483 & 4391 \\
10 & 0.60 & 3889 & 1688 & 5577 \\
11 & 0.70 & 6059 & 2065 & 8124 \\
12 & 0.80 & 7549 & 2570 & 10119 \\
13 & 0.90 & 9290 & 3120 & 12410 \\
14 & 1.00 & 11185 & 3739 & 18612 \\
15 & 1.10 & 14193 & 4419 & 21770 \\
16 & 1.20 & 16522 & 5248 & \\
\hline
\end{tabular}

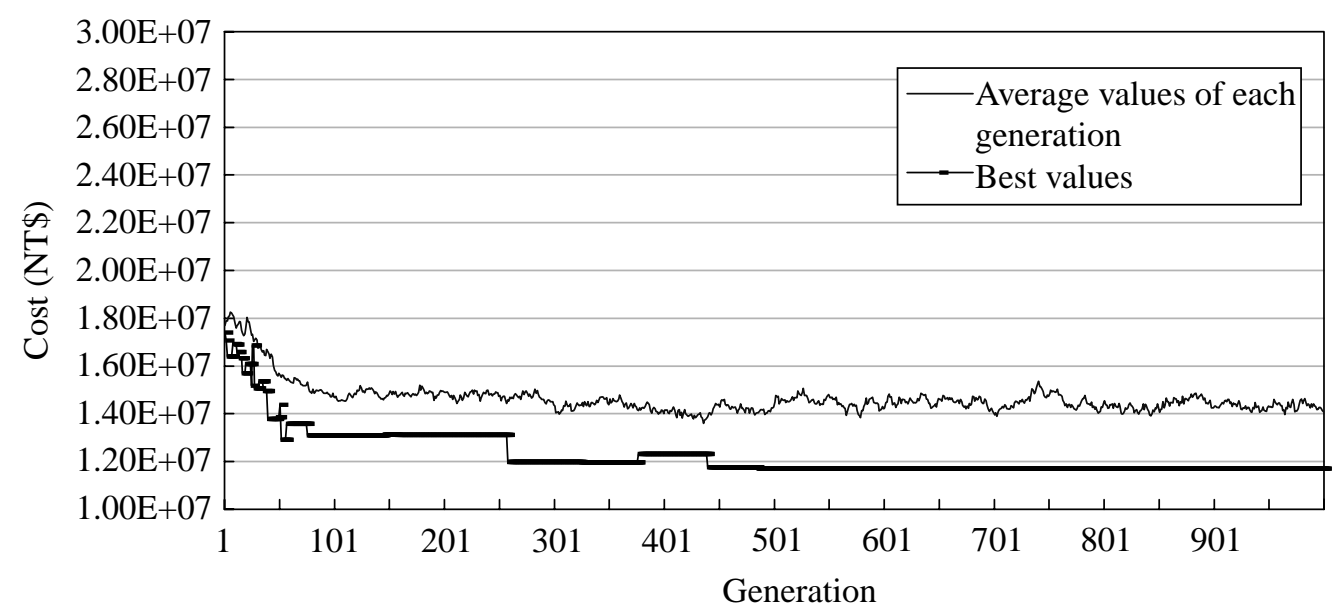

Fig. 2. The best value and average value of each generation.

to further investigation and readjustment.

(3) The number of generation:

The GA does not guarantee finding the global optimal solution. However, the GA can always find the near optimal solution with convergent generation. For limited time, 1,000 generation is preceded. Figure 2 is the best value and average value of each generation.

(4) The time of the solution:

The case at issue clearly indicates that, in principle, satisfactory solution may be attained. The solution time is mainly related to the number of evolving generations and the size of the population. The experiencebased practical design determines on the basis of flow volume the pipe diameter, water pressure, application environment and economic cost. While it takes three days to analyze by using the enumeration method, the GA method need only to take a couple of hours to analyze. The GA method undoubtedly saves a lot of time. Also, the GA analysis may handle a larger volume than other methods. The GA method is found to be more reliable.

(5) The value of the penalty function:

The purpose of the penalty function is to decrease the fitness degree of the solution that does not conform to the head limitation equation, and terminates its process of evolution. If the optimal solution is located at 
Table 3. Pipe and node characteristics for optimal design of Ruey-Fang system

\begin{tabular}{|c|c|c|c|c|c|c|}
\hline $\begin{array}{l}\text { Pipe } \\
\text { No. }\end{array}$ & $\mathrm{J} 1$ & $\mathrm{~J} 2$ & $\begin{array}{l}\text { Length } \\
\text { (M) }\end{array}$ & $\begin{array}{l}\text { Diameter } \\
\text { (M) }\end{array}$ & $\mathrm{C}$ & $\begin{array}{l}\text { Flow } \\
\text { (CMD) }\end{array}$ \\
\hline 1 & 1 & 2 & 320 & 0.5 & 100 & -9304 \\
\hline 2 & 2 & 3 & 520 & 0.5 & 100 & -8760 \\
\hline 3 & 2 & 4 & 1100 & 0.1 & 100 & -543 \\
\hline 4 & 4 & 7 & 380 & 0.1 & 100 & 657 \\
\hline 5 & 3 & 5 & 930 & 0.5 & 100 & -7420 \\
\hline 6 & 5 & 6 & 180 & 0.2 & 100 & -5485 \\
\hline 7 & 6 & 9 & 60 & 0.2 & 100 & -3417 \\
\hline 8 & 6 & 7 & 80 & 0.2 & 100 & -2067 \\
\hline 9 & 7 & 10 & 400 & 0.1 & 100 & -869 \\
\hline 10 & 10 & 17 & 420 & 0.1 & 100 & -329 \\
\hline 11 & 9 & 11 & 110 & 0.1 & 100 & -1500 \\
\hline 12 & 11 & 12 & 220 & 0.1 & 100 & -362 \\
\hline 13 & 12 & 13 & 120 & 0.2 & 100 & 1189 \\
\hline 14 & 9 & 14 & 130 & 0.1 & 100 & -1377 \\
\hline 15 & 14 & 15 & 210 & 0.1 & 100 & -514 \\
\hline 16 & 15 & 16 & 114 & 0.1 & 100 & -64 \\
\hline 17 & 11 & 14 & 90 & 0.1 & 100 & -136 \\
\hline 18 & 12 & 15 & 90 & 0.1 & 100 & -551 \\
\hline 19 & 13 & 16 & 90 & 0.3 & 100 & -10998 \\
\hline 20 & 16 & 17 & 150 & 0.3 & 100 & -10522 \\
\hline 21 & 5 & 8 & 190 & 0.1 & 100 & -1394 \\
\hline 22 & 8 & 18 & 390 & 0.1 & 100 & -394 \\
\hline 23 & 18 & 20 & 200 & 0.1 & 100 & 617 \\
\hline 24 & 18 & 19 & 280 & 0.1 & 100 & 189 \\
\hline 25 & 19 & 20 & 100 & 0.3 & 100 & 14717 \\
\hline 26 & 13 & 19 & 320 & 0.5 & 100 & 13987 \\
\hline Node & Joint & Source & $\begin{array}{l}\text { Demand } \\
\text { (CMD) }\end{array}$ & $\begin{array}{l}\text { Total head } \\
\text { (M) }\end{array}$ & $\begin{array}{l}\text { Elevation } \\
\text { (M) }\end{array}$ & $\begin{array}{l}\text { Operation } \\
\text { head }(\mathrm{M})\end{array}$ \\
\hline 1 & 1 & 1 & 9304 & 100.51 & 80.0 & 20.51 \\
\hline 2 & 3 & 0 & 0 & 100.19 & 78.0 & 22.19 \\
\hline 3 & 2 & 0 & -1340 & 99.73 & 75.0 & 24.73 \\
\hline 4 & 2 & 0 & -1200 & 85.73 & 70.0 & 15.73 \\
\hline 5 & 3 & 0 & -540 & 99.12 & 70.0 & 29.12 \\
\hline 6 & 3 & 0 & 0 & 93.26 & 68.0 & 25.26 \\
\hline 7 & 3 & 0 & -540 & 92.83 & 67.0 & 25.83 \\
\hline 8 & 2 & 0 & -1000 & 84.81 & 68.5 & 16.31 \\
\hline 9 & 3 & 0 & -540 & 92.45 & 67.0 & 25.45 \\
\hline 10 & 2 & 0 & -540 & 80.28 & 64.0 & 16.28 \\
\hline 11 & 3 & 0 & -1000 & 82.97 & 66.0 & 16.97 \\
\hline 12 & 3 & 0 & -1000 & 81.60 & 64.0 & 17.60 \\
\hline 13 & 3 & 0 & -1800 & 81.83 & 62.0 & 19.83 \\
\hline 14 & 3 & 0 & -1000 & 82.88 & 65.0 & 17.88 \\
\hline 15 & 3 & 0 & -1000 & 80.39 & 63.0 & 17.39 \\
\hline 16 & 3 & 0 & -540 & 80.36 & 61.0 & 19.36 \\
\hline 17 & 2 & 0 & -10850 & 78.10 & 60.0 & 18.10 \\
\hline 18 & 3 & 0 & -1200 & 81.99 & 66.5 & 15.49 \\
\hline 19 & 3 & 0 & -540 & 82.51 & 64.0 & 18.51 \\
\hline 20 & 2 & 1 & 15335 & 85.32 & 65.0 & 20.32 \\
\hline
\end{tabular}




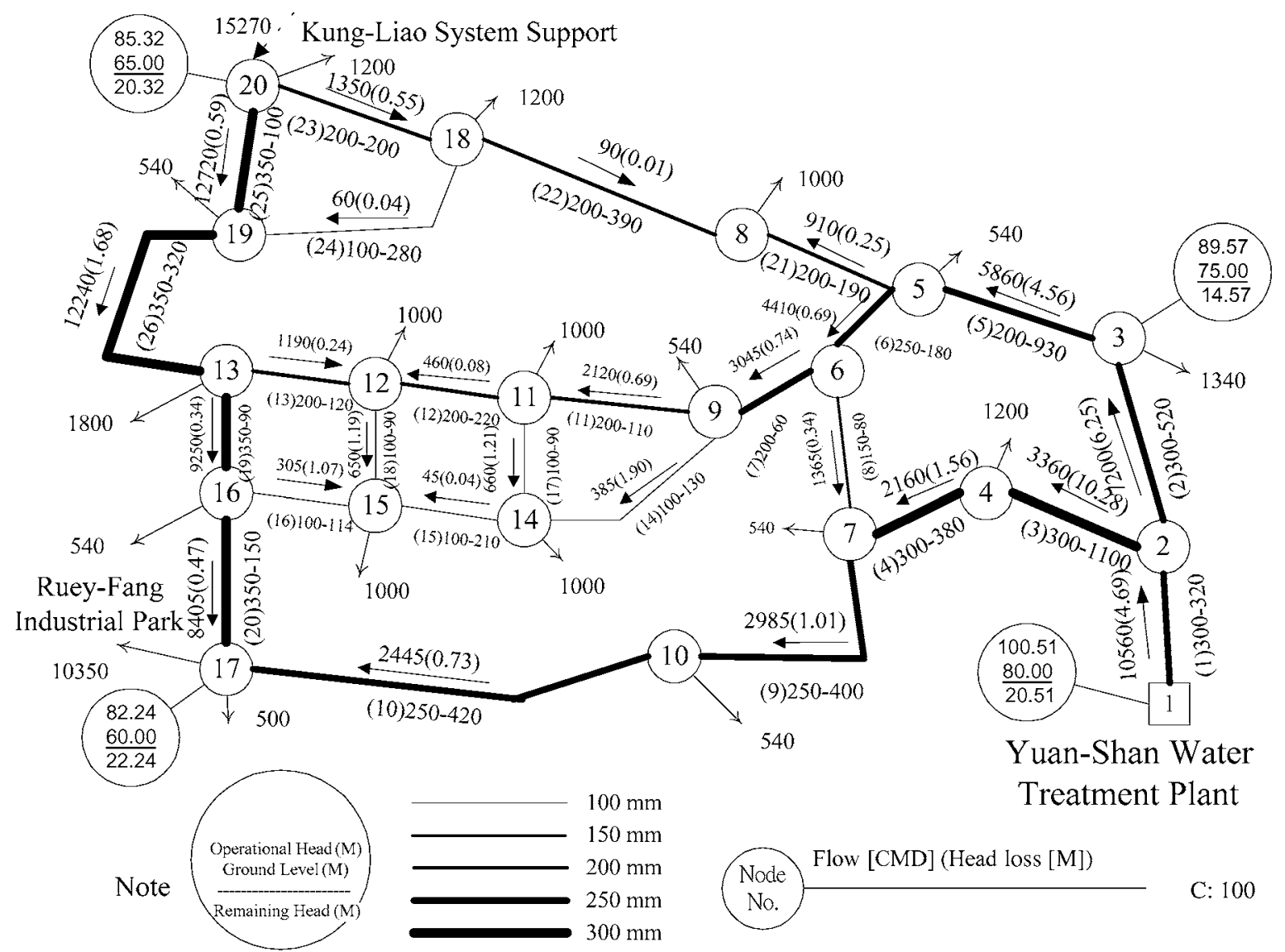

Fig. 3. System hydraulic analysis chart of Ruey-Fang system (Maximum hour).

the head limitation of $15 \mathrm{~m}$ or $40 \mathrm{~m}$, it may be the solution that does not correspond to the head limitation equation. The value of the penalty function has a great influence on the optimal solution.

(6) The bit number of the diameter:

It represents the size of the scale the system combination establishes. While 3 bits means there are eight optional commercial diameters for each pipe, 4 bits means there are sixteen optional commercial diameters for each pipe. The program will find the bit number automatically in each stage.

(7) The coding method:

The method of coding will affect the result of implementation. Both coding method of binary or grey will converge to the optimal solution, but the grey will find the solution efficiently.

\section{Evaluation of benefits}

(1) The method of Minimum Cost with water pipes is calculated to be $11,592,856$ NT\$ with remaining head 15.49 M-29.12 $\mathrm{M}$ as shown in Table 3. The system hydraulic analysis figure shows in Figure 4.
(2) By using the GA method, the simplified systematic solution, and the heuristic algorithm, this research designs a model that provides the greatest benefits to people. The model then draws up specifications to search for a feasible and economic project.

(3) It utilizes documents and the traditional drinking water system to design an optimal model and find a solution. By applying the GA and the concept of evolutionism the researchers set up an optimal model for the future.

\section{CONCLUSION}

1. When applying the GA to the pipe network systems of different sizes, we may get various solutions by proceeding evolution with diverse parameter values (e.g. the generation number of evolution, the population number of each generation, the penalty function factor, and the coding method, etc.) This research adopted a specific area to test and verify their respective proper parameter values. The finding shows the GA has the property of continuous improvement. Although it cannot guarantee a global optimal solution, it can 


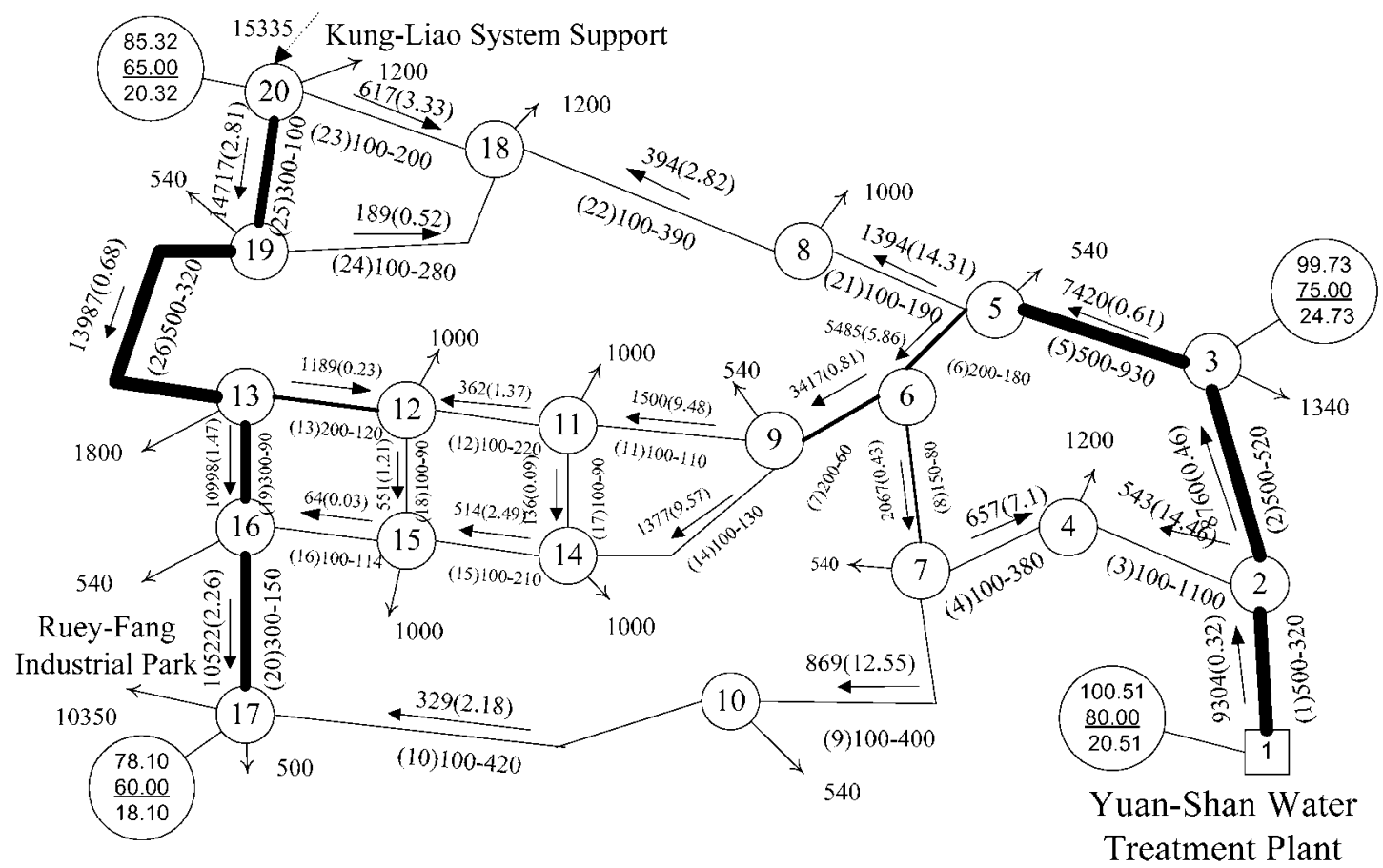

Fig. 4. Optimal solution with system hydraulic analysis chart of Ruey-Fang system.

always help us find the known optimal solutions for the case, and come up with the satisfactory solutions in a short time.

2. The result of the GA excellence selection shows that there is no need to seek a large number of combinations to obtain the excellence solutions. A few combinations are sufficient enough to enable us to obtain satisfactory results. Thus, we can prove that the GA is indeed an excellence searching method with high efficiency.

3. No matter we apply the GA or the boundary concealed enumeration method to solve their problems of the pipe network systems, we can always acquire the alternatives of the appointed number. These alternatives, which can be further analyzed and compared to enable us to have a better understanding of the whole system, provide us with meaningful information and also help to improve the quality of decision-making.

4. In addition to pipes, the optimal design with practical requirement of the pipe network systems should also take reservoirs, pumping stations, and relative auxiliary facilities into consideration. Meanwhile, system allocation should be closely connected to the geographic information system. Hence, the integration of an optimal method and geographical information system is the solution to the current planning and designing problems. Such an approach is worth further exploration.

\section{ACKNOWLEDGEMENTS}

The authors would like to thank the National Science Council of the Republic of China for its support of the research NSC 91-2211-E-159-001. The content of this paper is part of the results from that study.

\section{REFERENCES}

1. Brooke, A., Kendrick, D., and Meeraus, A., GAMS: A User's Guide, The Scientific Press, Redwood City, CA (1998).

2. Dandy, G.C., Simpson, A.R., and Murphy, L.J., "An Improved Genetic Algorithm for Pipe Network Optimization," Water Resour. Res., Vol. 32, No. 2, pp. 449-458 (1996).

3. Fujiwara, O., "A Modified Linear Programming Gradient Method for Optimal Design of Looped Water Distribution Network," Water Resour. Res., Vol. 23, No. 6, pp. 977-982 (1987).

4. Gessler, J., "Pipe network Optimization by Enumeration," Proceedings of Computer Applications for Water Resources, ASCE, New York, pp. 572-581 (1985).

5. Goldberg, D.E., Genetic Algorithms in Search, Optimization, and Machine Learning, Addison-Wesley Publishing Company, Inc., Boston, MA (1989).

6. Kessler, A. and Shamir, U., "Analysis of the Linear Programming Gradient Method for Optimal Design of Water Supply Networks," Water Resour. Res., Vol. 25, 
No. 7, pp. 1469-1480 (1989).

7. Lansey, K.E. and Mays, L.W., "Optimization Model for Water Distribution System Design," J. Hydraul. Eng., ASCE, Vol. 115, No. 10, pp. 1401-1418 (1989).

8. Liebman, J.S., Lasdon, L., Schrage, L., and Waren, A., Modeling and Optimization with GINO. The Scientific Press, Palo Alto, CA (1986).

9. Lin, B.L., Wu, R.S., and Liaw, S.L., "A Heuristic Approach Algorithm for the Optimization of Pipe Network Systems," Water Sci. Technol., Vol. 36, No. 5, pp. 219226 (1997).

10. Morgan, D.R. and Goulter, I.C., "Optimal Urban Water Distribution System," Water Resour. Res., Vol. 21, No. 5, pp. 642-652 (1985).

11. Pilar, M., Adela, G.G., and Jose, L.A., "Pipe Network
Systems Optimization Using a Modified Genetic Algorithm," Water Resour. Res., Vol. 35, No. 11, pp. 3467-3473 (1999).

12. Savic, D.A. and Walters, G.A., "Genetic Algorithms for Least-cost Design of Pipe Network Systems," J. Water Resour. Plan. Mgmt. ASCE, Vol. 123, No. 2, pp. 67-77 (1997).

13. Simpson, A.R., Dandy, G.C., and Murphy, L.J., "Genetic Algorithms Compared to Other Techniques for Pipe Optimization," J. Water Resour. Plan. Mgmt., Vol. 120, No. 4, pp. 423-443 (1994).

14. Wu, R.S. and Lin, Y.M., "The Optimal Design of Pipe Network System and Expanding Analysis," Water Supply Q., Vol. 14, No. 3, pp. 42-55 (1995). 